\title{
Demonstration of Multi-Star Wavefront Control for WFIRST, Habex, and LUVOIR
}

\author{
Dan Sirbu ${ }^{1,2}$, Ruslan Belikov ${ }^{1}$, Eduardo Bendek ${ }^{3}$, Chris Henze, Eugene Pluzhnik 1,2 \\ ${ }^{1}$ NASA Ames Research Center, Moffett Field, Mountain View, CA \\ ${ }^{2}$ Bay Area Environmental Research Institute, Moffett Field, Mountain View, CA \\ 3 Jet Propulsion Laboratory, California Institute of Technology, Pasadena, CA
}

\begin{abstract}
Coronagraphic instruments will enable direct imaging of dim planetary companions around nearby stars. The majority of nearby FGK stars are located in multi-star systems, including the Alpha Centauri stars, which may represent the best quality targets available for spectroscopic characterization due to their proximity and brightness. However, a binary system exhibits additional leakage from the off-axis companion star that may be brighter than the target exoplanet. Multi-Star Wavefront Control (MSWC) is a wavefront-control technique that allows simultaneous suppression of starlight of both stars in a binary system. MSWC would thus enable direct imaging of circumstellar planets in binary star systems such as Alpha Centauri.

MSWC is compatible with a wide suite of planned coronagraphic instruments for future space missions. As part of the technology demonstration efforts for MSWC, the first milestone results are presented here demonstrating (via computer simulations), high-contrast imaging capabilities of multi-star systems for several instruments including: [1] the upcoming WFIRST and its CGI demonstrator, [2] the HABitable Worlds EXplorer (Habex) mission concept, [3] the segmented Large UV/Optical/IR (LUVOIR) mission concept, and [4] a small-aperture Alpha Centauri direct-imager mission concept. For each of these missions, a baseline coronagraph option planned for the mission will be adopted and instrument baseline performance will be compared for single-star wavefront control.
\end{abstract}

Keywords: High-Contrast Imaging, Binary Stars, Wavefront Control, Multi-Star Wavefront Control, SuperNyquist Wavefront Control, WFIRST, Habex, LUVOIR

\section{INTRODUCTION}

The main challenge associated with the direct imaging of exoplanets is the suppression of both diffracted and scattered light from the host star so that the dim planetary companion can be imaged at sufficiently small angular separations and contrast ratios. ${ }^{1}$ The Wide Field Infrared Survey Telescope (WFIRST) mission will feature a technology-demonstrator coronagraph instrument (CGI) that will enable direct imaging of nearby star systems. Additionally, with their larger apertures the planned Habitable Exoplanet Imaging Mission (Habex) and the Large UV/Optical/Infrared Surveyor (LUVOIR) will be able to survey a larger number of nearby systems via direct imaging and characterize their planetary systems.

A coronagraph instrument consists of a set of internal optics that apply phase and amplitude changes at the image and pupil planes of the telescope to enable high-contrast imaging. Coronagraphs can suppress both diffraction due to the telescope aperture and additional wavefont errors introduced by surface aberrations which can potentially limit performance at several orders of magnitude above the needed contrast levels for direct imaging. All coronagraph-based high-contrast imaging systems designed for exoplanet imaging feature deformable mirrors (DMs) which enable a wavefront-control loop that removes aberration-induced speckles and remaining diffraction features (for example from primary mirror segmentation). This midspatial-frequency wavefront control loop creates high-contrast regions in the focal plane called dark holes.

Multi-star systems contain additional stellar components that may pose additional challenges for a coronagraphic instrument. The off-axis stellar components can introduce additional stellar leakage into the nominal 
dark hole which, depending on the specific form of the optical aberration power spectral density (PSD), separations between stellar components, and relative brightness can create a contrast floor limiting the ability of conventional coronagraphic systems to image targets with a stellar companion. Multi-star systems such as these actually represent a majority of nearby FGK stars. The Alpha Centauri stars are particularly attractive targets due to their proximity, and serve as a representative geometry for many other multi-star systems.

Multi-star wavefront control (MSWC) is a wavefront control technique that deals with the additional leakage from the off-axis star to enable direct imaging of exoplanets around binary star systems. The MSWC technique was introduced and extended elsewhere ${ }^{2,3}$ and we briefly review the basic principles and its operation modes (MSWC-0 and MSWC-s). This study builds on our previous investigation of multi-star imaging with WFIRST for stashades ${ }^{4}$ and coronagraphs ${ }^{5}$ outlining the applicability of MSWC to additional space mission configurations including the baseline Vector Vortex Cororonagraph Charge 6 (VVC6) on Habex and LUVOIR-B which were adopted by these mission concepts. These simulations represent Milestone \# 1 for the MSWC technology development program showing applicability of MSWC for planned NASA coronagraphic instruments. ${ }^{6}$ We first provide an update to the latest simulations showing MSWC applied to the WFIRST Shaped Pupil Coronagraph (SPC) in the Wide-Field Imaging Mode and showing operation for both Alpha Centauri A and B as targets and using official WFIRST optical specification. The Alpha Centauri stars are valuable scientific targets of their own for each of these missions, but also represent a canonical binary star geometry tests all aspects of MSWC. We then complete the MSWC Milestone \# 1 demonstrations for the same Alpha Centauri targets by applying MSWC to the Habex VVC6 and the LUVOIR-B VVC6. Finally, we consider MSWC for VVC6 and PIAA coronagraphs with a small-aperture direct imager.

\section{OVERVIEW OF MULTI-STAR WAVEFRONT CONTROL}

Nearly one half of FGK stars are located in multi-star systems as has been shown by surveys of nearby star systems. ${ }^{7,8}$ Of the nearest 20 FGK stars, 13 are located within multi-star systems. ${ }^{5}$ These nearby systems are particularly interesting targets for the WFIRST CGI on account of their proximity as their increased photon flux can result in better signal-to-noise ratios for imaging and characterization for finite observation times. Additionally, based on follow-up surveys of host stars of Kepler candidates it has been established that planets around binary stars are relatively common and may be as common as around single-stars. ${ }^{9}$ Within 10 pc, 42 out of 67 FGK stars are located in dynamical multi-star systems. ${ }^{10}$ On account of their larger apertures, Habex and LUVOIR will be able to directly image more distant targets. Within $20 \mathrm{pc}$, approximately 260 out of 510 nearby FGK stars are located in multi-star systems. Thus imaging binary star targets can significantly increase the scientific potential of a coronagraph instrument by: (1) increasing the number of targets available, (2) increasing the exoplanetary yield via additional targets (including high-quality, nearby targets), and (3) enabling binarysystem specific science. More details of the scientific questions enabled by imaging binary star systems with high-contrast imaging are detailed in a recent white paper. ${ }^{11}$

Direct imaging of multi-star systems is more challenging in comparison to a single star system because of light coming from off-axis star in addition to light from the central star. This off-axis starlight is mutually incoherent with the on-axis starlight and can be caused by both diffraction leakage and aberration-induced speckles. In general, except for the case of closely-separated binaries, the off-axis starlight tends to be dominated by high-frequency components from aberrations of the optical surface (especially from the telescope primary, but with contributions from other optical surfaces as well). The off-axis starlight must be suppressed over the same spatial region as the on-axis, central star to create a multi-star dark hole however this region would correspond to different spatial frequencies with respect to each of the stellar components.

The basic principle underpinning multi-star wavefront control is to use the wavefront control loop which is already present in all high-contrast imaging instruments to remove leakage from both stellar components simultaneously in the desired search region. This is possible only by using non-redundant modes on the deformable mirror to control for diffraction and speckles at different spatial frequencies for each of the stellar components. When the multi-star dark hole is located within the control limit with respect to each of the stars multi-star wavefront control is operating in the "sub-Nyquist regime" which we refer to as "MSWC-0" (with the name meaning control around the stellar core, or the zeroth order) and in principle there are no additional hardware requirements to enable this mode. However, for the case that the dark hole is outside of the DM's control limit 


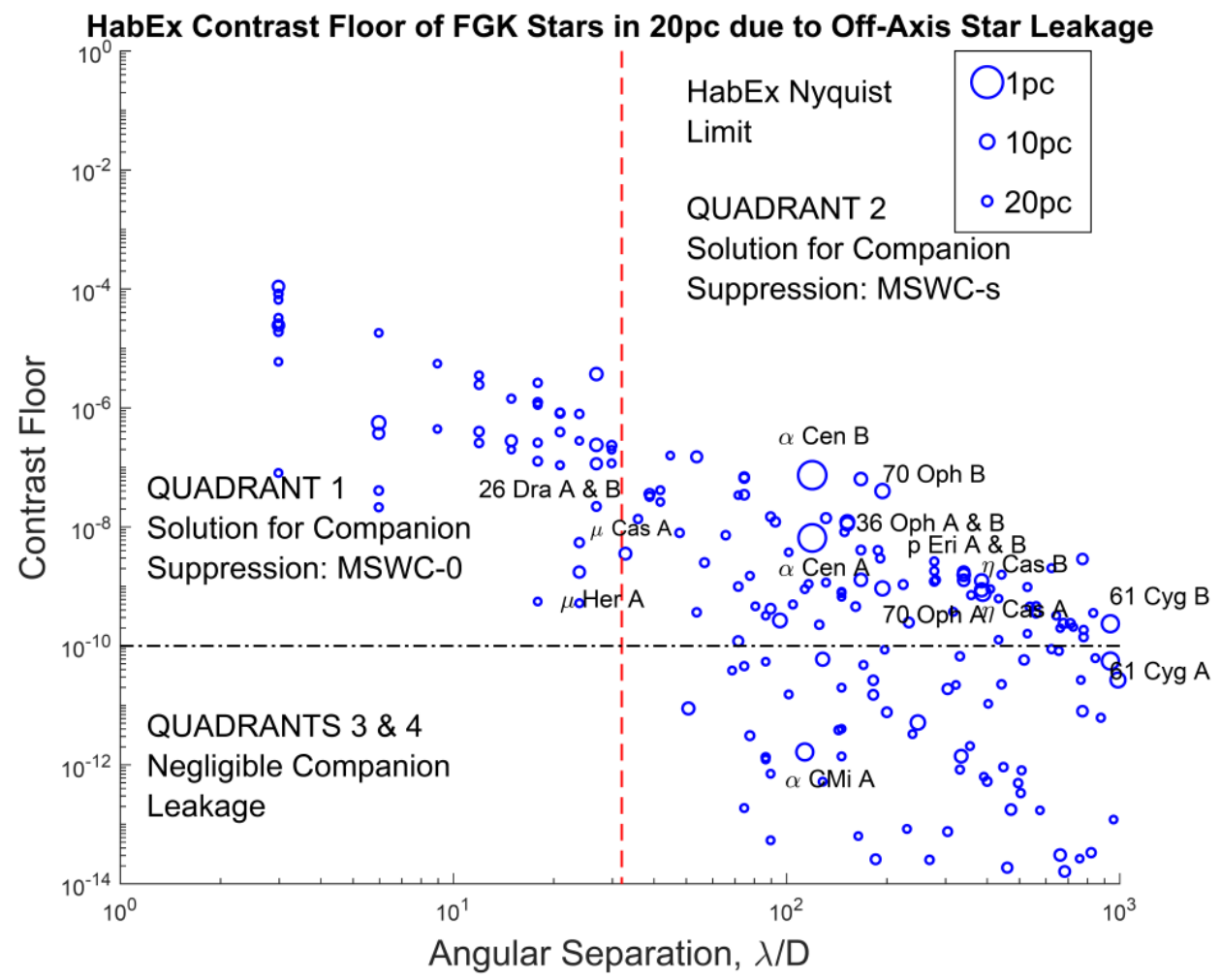

Figure 1: Computed contrast floor due to the off-axis leakage from the binary companion for WFIRST assuming a nominal PSD. Also shown is the 64 x 64 DM's Nyquist-limit and the 1e-10 raw contrast target limit.

for at least one of the stellar components a diffraction grating is needed to enable operation beyond the DM's nominal Nysuit limit. The basic idea is to use stellar replicas typically created by a diffraction grating which also effectively extend the DM control region in the "super-Nyquist" regime enabling "Super-Nyquist Wavefront Control" (SNWC). ${ }^{2}$ When the SNWC technique is combined with the principle of MSWC, any binary star separation can be controlled including those in the "super-Nyquist" regime and we refer to as "MSWC-s".

Several wavefront control algorithms have been developed to eliminate residual diffraction leakage and speckles due to aberrations in the optical train for a single on-axis star. These techniques include Electric Field Conjugation (EFC), ${ }^{12}$ Stroke Minimization, ${ }^{13}$ and traditional Speckle Nulling and can all be readily adapted for usage with MSWC. For example, EFC enables broadband wavefront control by extending the linearized system response matrix $\mathbf{G}$ for a monochromatic response via stacking additional system response matrices for each additional control wavelength to form a broadband response. MSWC can be implemented via the same technique by adding an additional set of simultaneous equations that correspond to the actuator response in the desired multi-star dark hole for each of the additional stars. The mathematical implementation of the MSWC extension to EFC is presented in a previous paper. ${ }^{3}$

The amount of leakage introduced by the off-axis companion is generally dominated by aberrations on the optical surface of the telescope primary. Aberrations on the secondary mirrors as well as additional imaging optics inside the coronagraph instrument may also be contributing additional leakage. The intensity of the offaxis speckles is determined by three main factors: (1) the brightness ratio between the target star and its off-axis companion, (2) the angular separation of the binary companion, and (3) the particular power spectral density (PSD) distribution corresponding to the spatial frequencies across the region of interest. The first two factors are dependent on the stellar parameters of the binary components, while the third factor is dependent on the 
imaging system optical quality.

In this paper, for the binary stellar components we utilize the Alpha Centauri stellar geometry. With a projected separation of 7 arcseconds in 2027 , we choose this separation as being directly relevant for the WFIRST mission. We use this same separation as well for the Habex and LUVOIR simulations in order to directly compare the effect of the aperture size for the same stellar geometry. The Vmag difference between the two stars corresponds to Alpha Centauri A being approximately 3 times brighter than Alpha Centauri B.

We generate PSD realizations in PROPER ${ }^{14}$ using the following general formula:

$$
\operatorname{PSD}(f)=\frac{E_{\mathrm{res}}}{1+\left(f / c_{l}\right)^{f_{p}}}
$$

In the above, the spatial frequency is measured in cycles per meter. We set $E_{\mathrm{res}}=20 \mathrm{~nm}$ RMS corresponding to the total amount of expected aberration across the primary. The spectral envelope is relatively flat and then decays following the knee. The rate of decay at high frequencies is set by $f_{p}=2.5$ corresponding to a $f^{-2.5}$ power law. Finally, we set the correlation length parameter $c_{l}$ to correspond to 8 cycles/aperture which controls the length of the flat portion of the spectral envelope before the knee. Note that this is an updated PSD specification compared to the specification assumed in our previous WFIRST-focused paper. ${ }^{5}$ The WFIRST primary PSD has also been adopted as a canonical example for the Habex study, and as such we will utilize the same PSD specifications for Habex. The same PSD will also be applied to the overall LUVOIR aperture aberrations, though the specific PSD for LUVOIR has been the subject of recent studies particularly focusing on the mirror aberration segmentations. ${ }^{15}$

In Figure 1, we plot the computed leakage for all FGK stellar targets within 20 pc due to their binary companion using the 4-m Habex aperture and the PSD specification above. The stellar geometry is obtained by cross-referencing targets with the Washington Double Star (WDS) catalog, obtaining the most up-to-date stellar separations and visual magnitude ratios, and determining the appropriate primary and secondary components from the list of companion entries. The vertical red line indicates the Nyquist limit for an assumed pair of $64 \times 64$ DMs. All stars above the $10^{-10}$ raw contrast limit must have their off-axis leakage suppressed to enable direct imaging at the baseline contrast level. A few stars are labelled for reference. In particular, the Alpha Centauri A is showing a contrast floor of 2.8e-8 induced by Alpha Centauri B. Conversely, Alpha Centauri B has a shallower contrast limit of 2.5e-7 induced by the brighter Alpha Centauri A star. To reach the same raw contrast limit in the same dark zone region is more challenging for Alpha Centauri B (on-axis) than for Alpha Centauri A (on-axis) since additional off-axis leakage must be removed. Note that the Alpha Centauri stars are representative targets for operation in the MSWC-s regime and will serve as canonical examples for MSWC operation in addition to being prime science targets themselves.

For all the simulated MSWC scenarios below we use a common, compact layout swapping in coronagraph and pupil elements that represent the configuration under consideration. All scenarios feature 2-DM control with Fresnel propagation between the DMs which models the Talbot effect. Phase aberrations following the PSD specification above are assumed on the primary which represents the system pupil. Although no amplitude errors are specifically introduced, due to the Talbot effect there is phase-to-amplitude mixing of errors and thus the 2-DM system must take into account both phase and amplitude control as well as chromaticity effects. Additional level of fidelity planned in the future would include the addition of sensor noise in combination with wavefront-reconstruction in the noise-limited regime while these simulations assume perfect knowledge of the wavefront. However, these simulations serve as a demonstration that the coronagraphic modes surveyed in this paper are compatible with MSWC at the baseline contrast levels required for scientific operation.

\section{WIDE FIELD INFRARED SURVEY TELESCOPE IN WIDE-FIELD IMAGING MODE}

The WFIRST telescope feature a 2.4-m primary with a large central obscuration and thick struts. The WFIRST CGI instrument features three different coronagraphic modes using a single Lyot-style architecture:

- Hybrid Lyot Coronagraph (HLC) Narrow-Field Imaging Mode featuring a 360-degree dark hole spanning 3-9 $\lambda / D$ (fed to EMCCD camera) 


\section{Single-Star Wavefront Control}

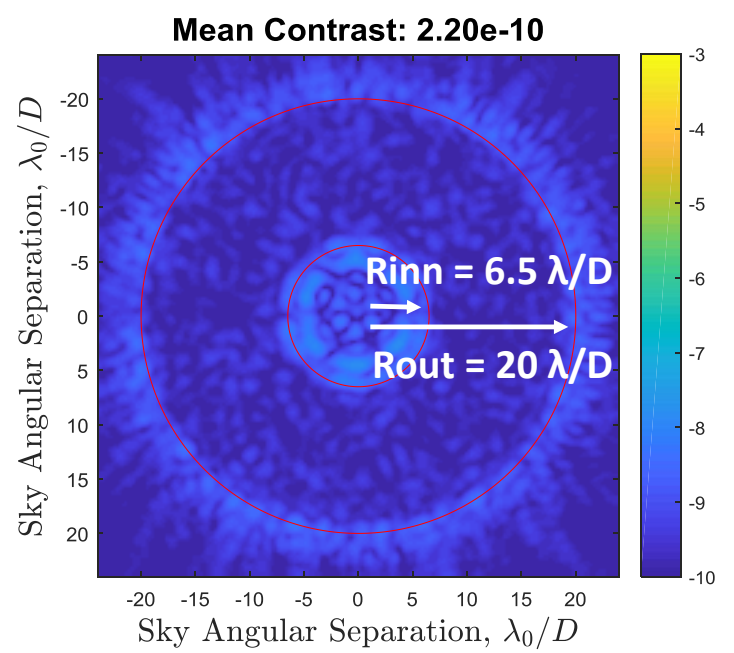

(a)

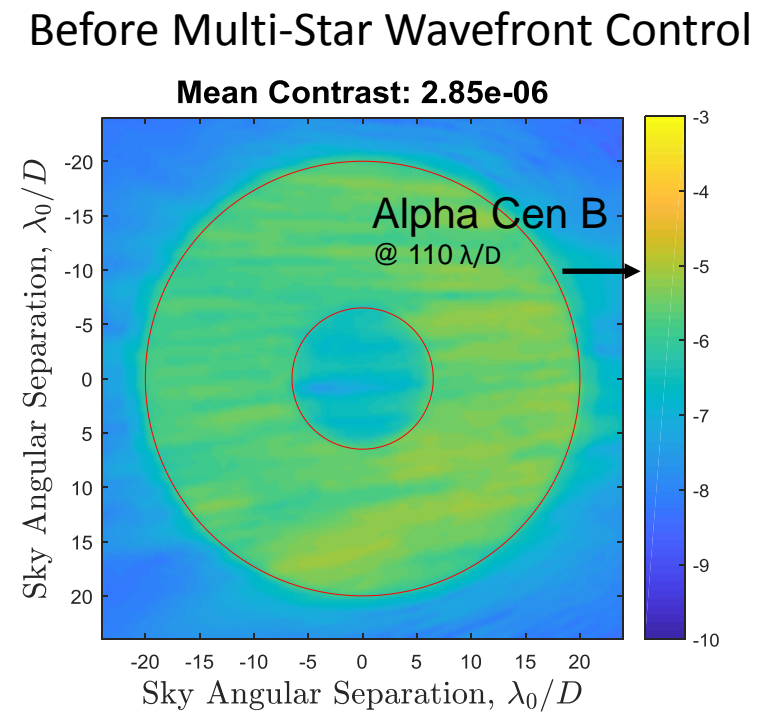

(b)

Figure 2: Effect of companion's star leakage on WFIRST SPC's Wide-Field Imaging Mode: (a) Dark hole created using traditional single-star wavefront control in $10 \%$ band showing a contrast level below the 1e-9 target, (b) Keeping the single-star wavefront control settings on the DM, but adding the off-axis source introduces a contrast floor due to the off-axis leakage.

- Shaped Pupil Coronagraph (SPC) Spectroscopy Mode featuring a double-sided 120-degree dark hole spanning 3-9 $\lambda / D$ (fed to the IFS)

- Shaped Pupil Coronagraph (SPC) Wide-Field Imaging Mode featuring a 360-degree dark hole spanning 7-20 $\lambda / D$ (fed to EMCCD camera)

All three CGI instruments have currently baselined a pair of 48 x 48 Xinetics DMs for the WFC loop that enables high-contrast imaging with a double-sided dark hole. We have chosen to demonstrate MSWC in simulation with the SPC in the Wide-Field Imaging Mode because this mode features the widest field of view extending out to $20 \lambda / D$. For the Alpha Centauri stars in particular because of its proximity the habitable zone spans a very large area. The field of view of the Wide-Field Imaging mode allows capturing the entire habitable zone of Alpha Centauri B. A planet in the habitable zone of Alpha Centauri A would be visible at quadrature nearer the inner working angle. The WFIRST SPC Wide-Field mask was originally designed for a mean contrast of $7.8 \times 10^{-10}$ in $10 \%$ around $575 \mathrm{~nm} .{ }^{16}$ Experimental performance was recently verified in the $820 \mathrm{~nm}$ channel in a vacuum test at HCIT to a level of $3.5 \times 10^{-10} .^{17}$

Since DMs are available on all planned coronagraph modes, MSWC can be applied algorithmically for any of these modes. However, the Xinetics DMs feature a very low amount of quilting after polishing and thus the natural quilting that is present on a DM surface may be insufficient with these particular DMs to create a multi-star dark hole when operating in the MSWC-s regime (which is needed for the majority of possible targets including the Alpha Centauri stars). To enable MSWC-s a diffractive pupil is needed to create diffractive orders $;^{2}$ a possible solution for this would be to use a diffractive pupil that is identical to the SPC Wide-Field Mask using superimposed non-reflective dots which would create diffraction orders. A general purpose diffractive mask could be created that features diffraction orders that cover the entire usable field of view with higher orders used for more widely-separated stars. Alternatively, a set of masks that are optimized for specific targets to place diffractive orders only where needed could be used instead. For the simulations here, we have used a diffractive pupil with a uniformly distributed set of dots that cover about $1 \%$ of the usable area thus having a small impact on overall throughput, with spacing that matches the DM actuator spacing. ${ }^{5}$

To compare MSWC performance, we first use the modelled WFC loop to generate a single-star dark hole for the WFIRST SPC Wide-Field Imaging mode at a performance level below the targeted raw contrast limit 


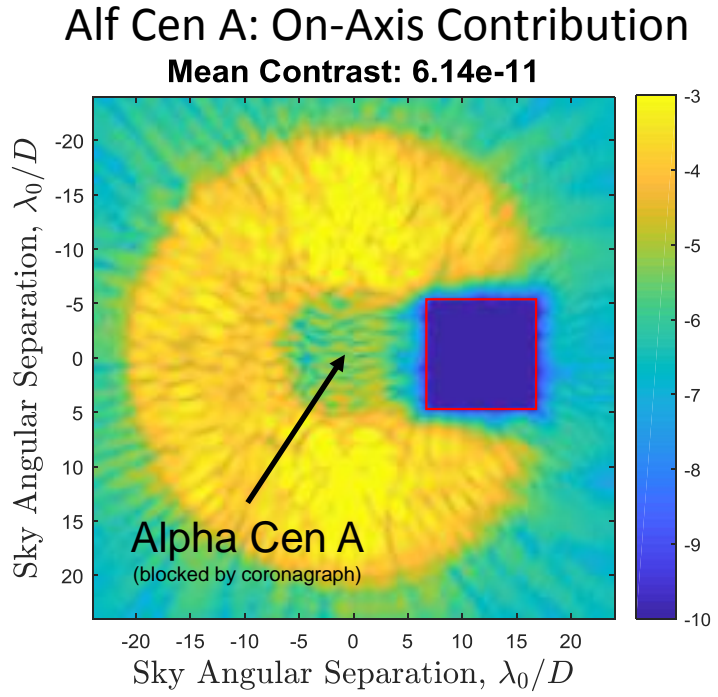

(a)

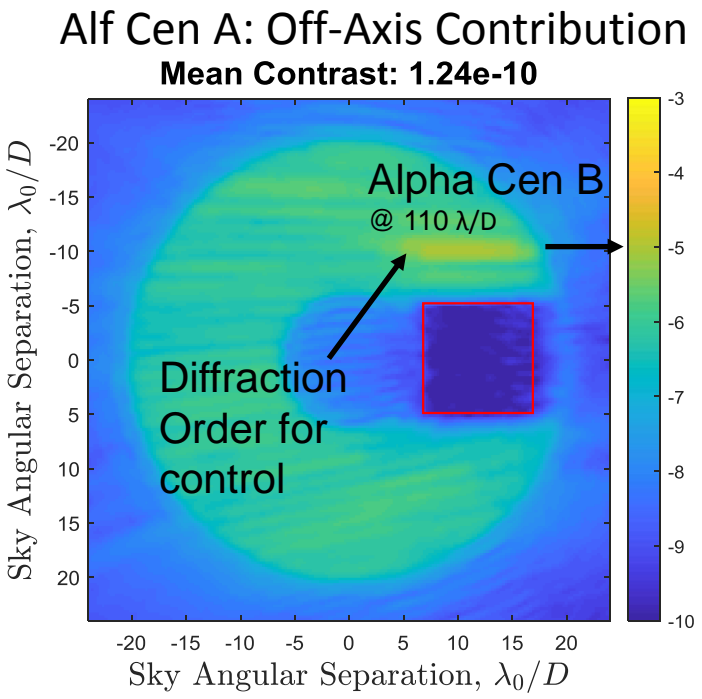

(b)

Figure 3: MSWC settings are applied on the DMs for WFIRST SPC Wide-Field mask to control both the on-axis source and off-axis simultaneously in a $10 \%$ band: (a) On-axis contribution from Alpha Centauri A only, (b) Off-axis contribution from Alpha Centauri B only.

level of 1e-9 and similar to that experimentally demonstrated at HCIT. We then maintain the wavefront control loop's optimized DM settings and introduce a second off-axis star to demonstrate the contrast floor expected due to the off-axis star. This simulated scenario is shown in Figure 2. In Figure 2a, the single-star dark hole is shown to have a mean contrast performance of $2.20 \mathrm{e}-10$ in a $10 \%$ band centered around $575 \mathrm{~nm}$ and spanning a double-sided 360-degree dark hole from 7-20 $\lambda / D$. For a multi-star imaging scenario, the single-star wavefront control solution shown above would suppress speckles from the on-axis star. However, performance would be limited by the off-axis star. This is shown in Figure 2b, where the leakage induced by the off-axis star limits contrast at the $2.85 \mathrm{e}-06$ level in a $10 \%$ band. The off-axis star is located at $110 \lambda / D$ off-axis, which is consistent with the separation between the Alpha Centauri stars.

Next, we use MSWC-s to remove speckles simultaneously from both the on-axis and off-axis stars for this configuration as shown in Figure 3. We use a $10 \times 10 \lambda / D$ multi-star dark zone located between 7 and $17 \lambda / D$ along the horizontal axis and between -5 to $5 \lambda / D$ along the vertical axis in the same $10 \%$ band around 575 $\mathrm{nm}$. The maximal dark zone area possible with MSWC is half the dark zone size controllable with single-star wavefront control. In practice, additional stroke required on the DM may limit the dark zone size to smaller than the theoretical maximum due to chromaticity and nonlinear effects that must also be controlled. In this simulated scenario, we use the Alpha Centauri A star on-axis and the B-star off-axis. The smaller dark hole size of the on-axis star is controlled well by MSWC with a mean contrast of 6.14e-11 as shown in Figure 3a. The off-axis dark zone, however, is limiting contrast at 1.24e-10 as shown in Figure 3b. Also indicated is the third diffraction order of the diffractive pupil used for controlling the high-frequency speckles of the off-axis star. This diffractive order is elongated to the chromaticity of the off-axis star in $10 \%$ band. The diffractive order has a contrast at the 1e-3 level in monochromatic light (though its broadband elongation distributes light at a level closer to 1e-4 contrast). MSWC imposes larger strokes than are typical of single-star EFC, but these settings were obtained at overall mild levels and below the more aggressive stroke levels already demonstrated with the Hybrid-Lyot Coronagraph for removing the WFIRST strut features (we expect this level of MSWC stroke when used with the HLC would represent an addition to the stroke required to control diffraction from the struts, but would be a small addition to the overall stroke required). DM-1 features actuators with $35.8 \mathrm{~nm}$ RMS and 274 $\mathrm{nm}$ P-V, while DM-2 features actuators with $34.9 \mathrm{~nm}$ RMS and $297 \mathrm{~nm} \mathrm{P-V}$. These correspond to an on-axis Strehl Ratio of 0.71 , and thus represent a relatively mild loss of throughput.

The combined multi-star dark hole is shown in Figure 9. Both the Alpha Centauri stars are set as on-axis 


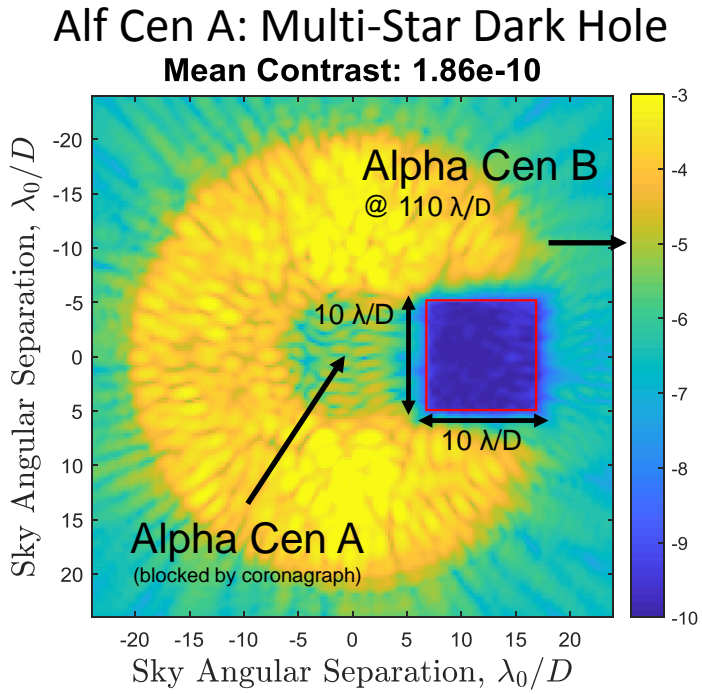

(a)

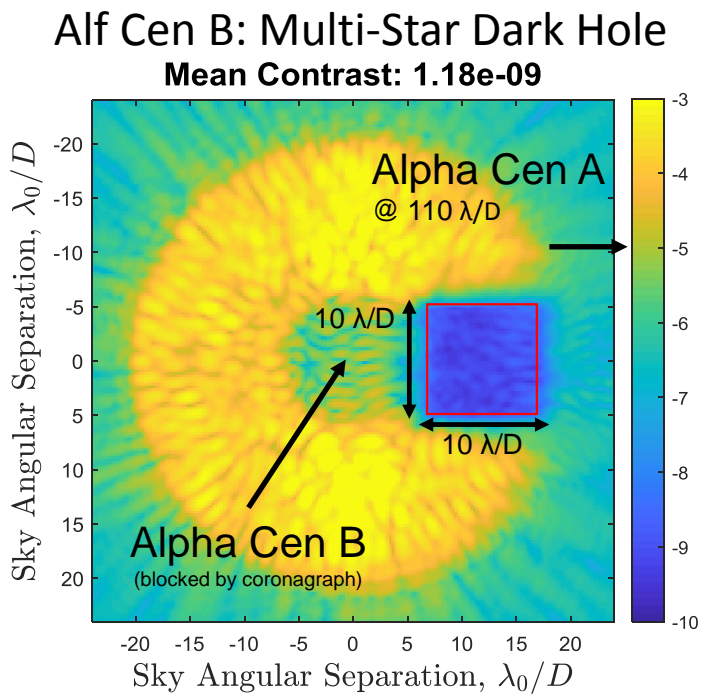

(b)

Figure 4: Multi-Star Dark holes when MSWC settings are applied on the WFIRST SPC DMs in 10\%: (a) Multi-star dark hole with Alpha Centauri A as the target star, (b) Multi-star dark hole with Alpha Centauri B as the target star.

targets using the same MSWC-generated DM settings with the multi-star dark zone indicated for Alpha Centauri A in Figure 4a and for Alpha Centauri B in Figure 4b. When maintaining the same DM settings, the mean contrast for observing Alpha Centauri A is 1.86e-10 while for B it is 1.18e-09. The multi-star dark zone is shallower for Alpha Centauri B since the contrast is limited by the off-axis star's leakage. Due to the 3 times brightness ratio between the stars, this contrast will be at a contrast level approximately 9 times greater when the brighter Alpha Centauri A star is located off-axis.

Finally, we explore the possibility of using MSWC for the WFIRST SPC in a $15 \%$ band as shown in Figure 5. The on-axis and off-axis contributions are shown in Figure 5a and Figure 5b respectively. Their combined dark zone is shown in Figure 5c with a mean contrast of 7.89e-10. In general the dark hole size and the bandwidth can be traded off against each other, and this trend also applies to MSWC. As a starting point for an exploration at wider bands, we have reduced the dark hole size to $7 \times 7 \lambda / D$. The average strokes on the DMs for this wider band configuration are actually lower than for the larger dark zone demonstrated above, with on-axis Strehl Ratio of 0.81 . This suggests that it may be possible to trade-off additional stroke to increase the dark hole size closer to $10 \times 10 \lambda / D$.

Compared to the MSWC results presented previously for WFIRST, ${ }^{5}$ these results focused on 2-DM control. Additionally, these results are with realistic levels of aberrations expected from the WFIRST primary PSD and show contrast performance which enables the target raw contrast of 1e-9 for both the Alpha Centauri stars (including the more challenging case of Alpha Centauri B).

\section{HABITABLE WORLDS EXPLORER WITH VECTOR VORTEX CORONAGRAPH}

The Habex mission study included a number of architectures. We choose to focus on the Habex-A architecture which features a 4-m primary with a monolithic mirrror. The Habex-A architecture features both a starshade and a VVC-6 coronagraph with dual-DM control featuring $64 \times 64$ actuators. In this section, we focus on MSWC with the VVC-6 architecture though it is worth noting that the Habex-A study illustrated the option of maintaining compatibility with usage of a hybrid DM and starshade architecture which would enable binary star imaging with a starshade as well. The primary PSD specification is assumed to be the same as for WFIRST as discussed in Section 2. To enable MSWC-s, we assume that a Boston MEMS DM is available on Habex with mild natural quilting. Although a $64 \times 64$ quilting model is not currently available, we have extrapolated the quilting model 


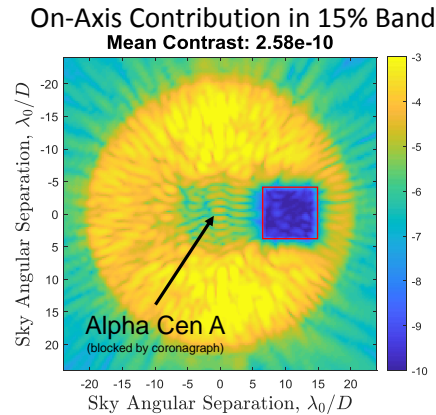

(a)

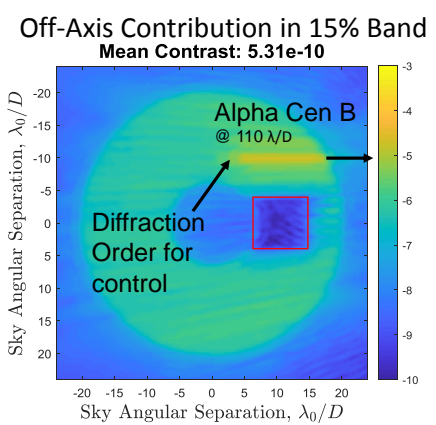

(b)

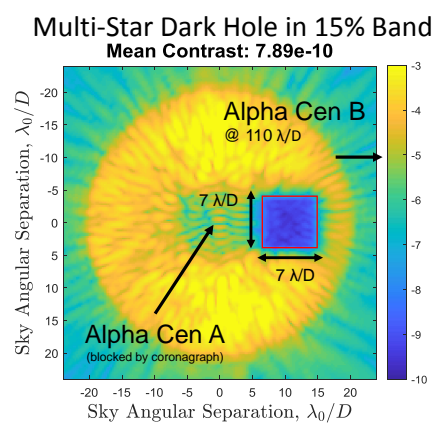

(c)

Figure 5: Multi-Star Dark holes when MSWC settings are applied on the WFIRST SPC DMs in 15\%: (b) On-axis contribution from Alpha Centauri A, (b) Off-axis contribution from Alpha Centauri B, (c) Combined multi-star dark zone

exhibited by a $32 \times 32 \mathrm{DM}$ used in the Ames coronagraph testbed based on high-resolution interferometric images provided by Boston Micromachines and detailed elsewhere. ${ }^{18}$

The VVC-6 design for Habex features a dark hole spanning 2.5-15 $\lambda / D$ in a 360-degree region when operating in single-star coronagraphic imaging mode. As a first step to demonstrate the baseline VVC-6 Habex design performance, we use EFC to generate such a double-sided dark zone to a contrast level below the 1e-10 raw contrast required. This is shown in Figure 6a. We then add an off-axis star corresponding to Alpha Centauri B at $200 \lambda / D$ separation while keeping the same settings on the DM that generated the single-star dark zone. The contrast floor introduced by the off-axis leakage is shown in Figure 6b, with mean contrast degrading across the dark hole to 2.76e-08. This is a milder contrast floor than for the WFIRST case even though we use the same PSD specification - this is primarily because the telescope diameter for Habex is increased compared to WFIRST resulting in a larger stellar separation in terms of $\lambda / D$ and thus a milder leakage level due to the $f^{-2.5}$ optical aberation high-frequency fall-off spectral envelope. In addition, there are second-order diffraction effects due to the difference in the pupil geometry between WFIRST and Habex as well as the specific coronagraph.

Now that that the baseline Habex performance with an off-axis star has been determined, we apply MSWC-s to remove speckles from the off-axis star simultaneously with the on-axis star. In Figure 7 we show the control of the off-axis source's speckles at high-frequencies using the third-order diffraction spot (with a 64 x 64 DM, the spacing between the orders is now correspondingly increased). In $10 \%$ band, this is elongated from chromaticity even more than for the WFIRST case before (compare with Figure 3b). Thus even though the speckle contrast is lower this is mostly compensated by the pronounced chromaticity. Overall, the DM strokes are actually lower than for the WFIRST case with the on-axis Strehl ratio at 0.78. Figure 7 shows the chromatic behaviour inside the MSWC dark-zone outlining the contributions from the on-axis and off-axis dark zones scanning through wavelength. In this plot the two binary components are assumed equal brightness. The optimized wavelengths are clearly visible with degradation of contrast in-between the wavelengths used for control. The mean, broadband contrast level of $5.81 \mathrm{e}-11$ is also indicated.

The final MSWC dark holes are shown in Figure 8 when observing either of the Alpha Centauri stellar components. In this case, the same MSWC settings are maintained on the DM and the observed component is swapped. As before, because of its brightness the leakage from Alpha Centauri A when off-axis is more difficult to remove than the leakage from Alpha Centauri B when off-axis. This is reflected in the deeper contrast of 2.76e-11 achieved when Alpha Centauri A is observed on-axis in Figure 8a compared to the mean contrast of 1.50e-10 when Alpha Centauri B is observed on-axis in Figure 8b.

\section{LARGE UV/OPTICAL/INFRARED SURVEYOR WITH VECTOR VORTEX CORONAGRAPH}

The LUVOIR mission study included two main architectures. The LUVOIR-A architecture uses a large, on-axis 15-m telescope with struts and segmentation. This architecture has baselined the Apodized Lyot Coronagraph 


\section{Single-Star Wavefront Control}

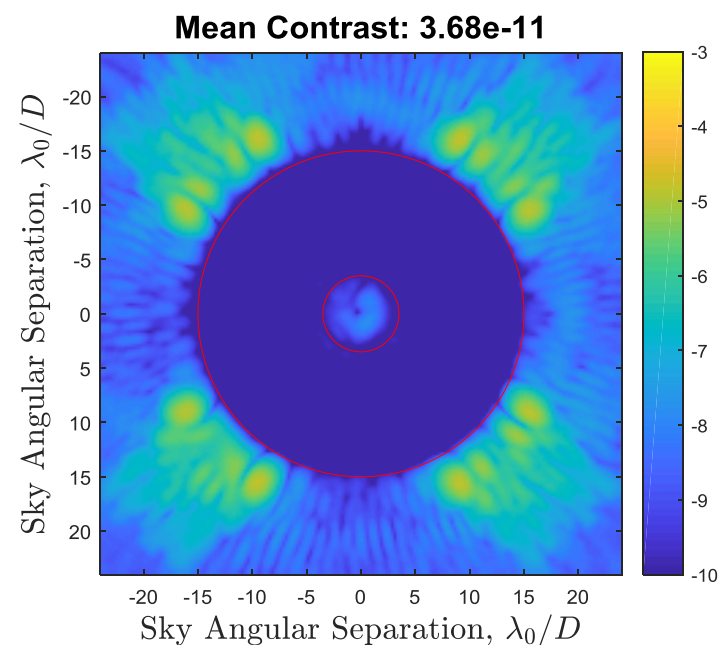

(a)

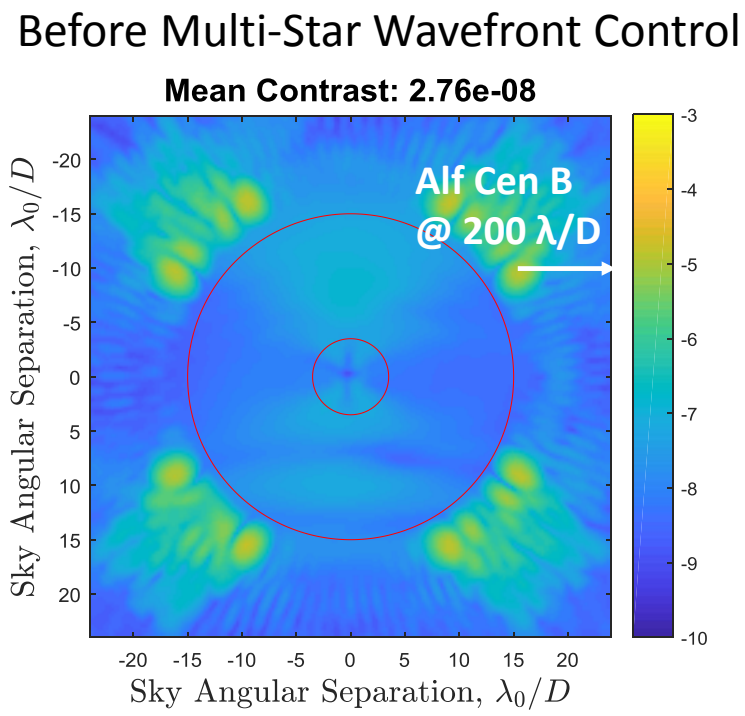

(b)

Figure 6: Baseline performance for the VVC-6 Habex coronagraph in a 10\% band: (a) Single-star wavefront control showing a double-sided dark hole with contrast below the required 1e-10, (b) Keeping single-star wavefront control settings on the DM, but adding the off-axis source limits introduces a contrast floor due to the off-axis leakage at $2.76 \mathrm{e}-08$.

\section{Alf Cen A: Off-Axis Contribution}

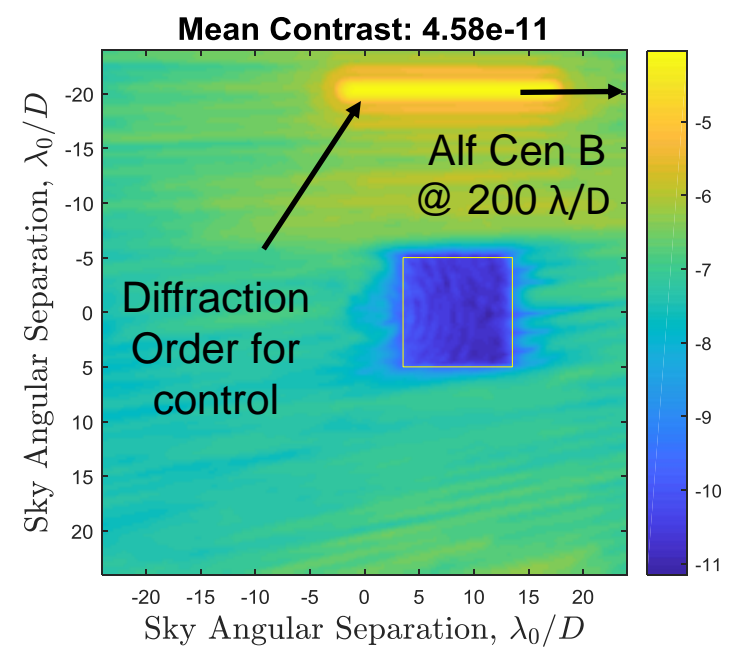

(a)

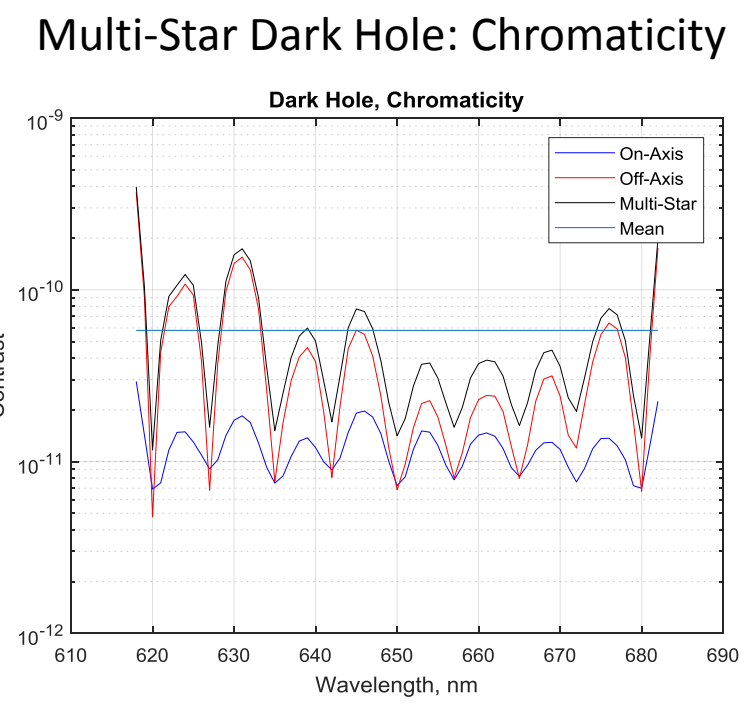

(b)

Figure 7: MSWC-enabled dark hole for Habex in $10 \%$ band with equal-brightness components: (a) The leakage from the off-axis source dominates the dark hole residual but is now controlled to a level below 1e-10, (b) Chromaticity analysis of multi-star dark zone showing the mean contrast and the incoherent contributions for the on-axis and off-axis source.

(APLC) which can effectively deal with the on-axis obstruction. The LUVOIR-B architecture uses a smaller 8-m circumscribed telescope which is off-axis and thus has no struts or obstruction, but does feature segmentation. The LUVOIR-B coronagraph is very similar to Habex-A - a VVC-6 coronagraph dealing with the effects of the inscribed aperture while the dual-DM system is used to remove the effects of the primary segmentation. We chose to focus on the LUVOIR-B architecture for the simulated demonstration in order to more easily assess for the effect of aperture sizing rather than the specific choice of coronagraph (by minimizing differences in comparison with the previous Habex result). In both cases, LUVOIR is baselining a larger $128 \times 128$ dual DM system with 
Alf Cen A: Multi-Star Dark Hole

Mean Contrast: 2.76e-11

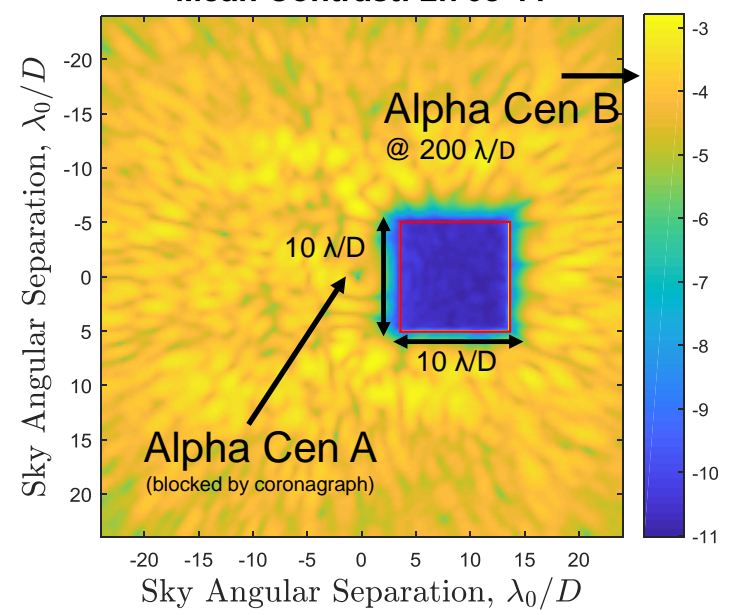

(a)

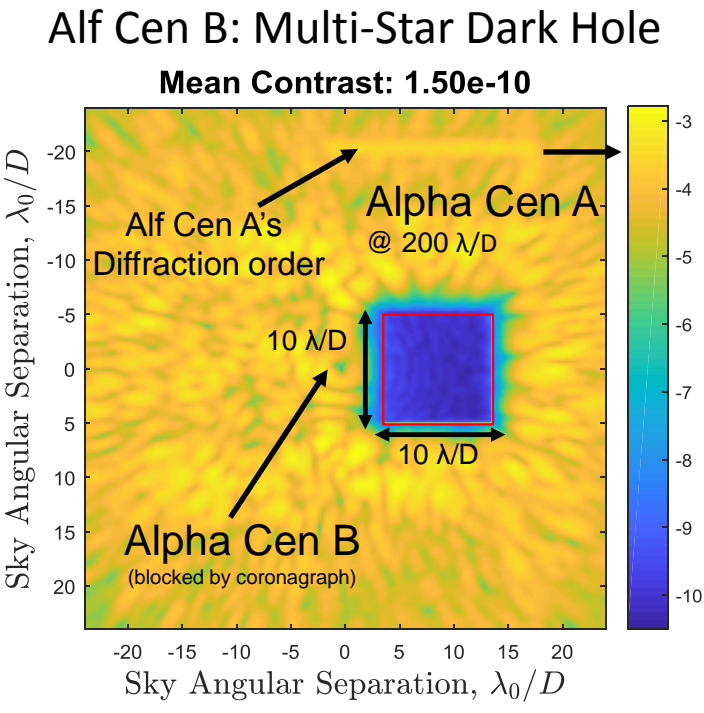

(b)

Figure 8: Final MSWC dark holes in 10\% band for with Habex VVC-6 and with contrast within the 1e-10 target raw contrast when observing: (a) Alpha Centauri A, (b) Alpha Centauri B.

three different channels for simultaneous characterization at different bands.

We started by reproducing comparable performance for the LUVOIR-B VVC-6 design baseline which features a dark hole spanning 2.5-15 $\lambda / D$ in a 360-degree region and demonstrating that we can use the DMs to remove the segmentation diffraction features. To obtain this baseline performance, EFC generates a double-sided dark zone to a contrast level below the 1e-10 raw contrast required. This is shown in Figure 9a. An off-axis star corresponding to Alpha Centauri B is introduced at $400 \lambda / D$ (corresponding to twice the separation for Habex shown in Figure $6 \mathrm{~b}$ due to the doubling in telescope diameter) separation while keeping the same settings on the DM that generated the single-star dark zone. The contrast floor introduced by the off-axis leakage is shown in Figure 9b, with mean contrast degrading across the dark hole to 2.20e-09 (which is again milder than either WFIRST or Habex as expected on account of the telescope diameter while using the same PSD).

The final MSWC dark holes for the LUVOIR-B VVC-6 are shown in Figure 10 when observing either of the Alpha Centauri stellar components. When observing Alpha Centauri A, a mean contrast across the multi-star dark hole of $6.92 \mathrm{e}-11$ is obtained. Figure 8a compared to the mean contrast of 1.08e-10 when Alpha Centauri B is observed on-axis in Figure $8 \mathrm{~b}$.

\section{ALPHA CENTAURI DIRECT IMAGING CONCEPT}

As a final case study, we study a small-mission concept with a monolithic (off-axis) primary of 0.4-m diameter and operating in a $15 \%$ blue-band around $430 \mathrm{~nm}$. This mission concept (ACEND) is focused on imaging Alpha Centauri exclusively and considered both a VVC-6 and a classical PIAA (Phase-Induced Amplitude Apodization) coronagraph $^{19}$ with MSWC to enable suppression of the off-axis star. The PIAA configuration uses both forward and inverse PIAA optics which is helpful for sharpening the off-axis aberrations and may help decrease the stroke requirements on the DM. In this way, the PSF sharpness between the two coronagraphs is very similar. The coronagraph instrument envisioned a single DM - due to the smaller aperture, a modest dark size would obtain the entire one-sided habitable dark beyond the coronagraph's inner working angle of $2.3 \lambda / D$ and extending out to $5 \lambda / D$.

Although MSWC results for this case are not part of the formal MSWC milestone, we include these here as part of an exploration of the effect of aperture sizing on binary companion and as an opportunity to demonstrate MSWC with an additional coronagraph architecture (PIAA). With a smaller aperture, the stellar separation between the Alpha Centauri stars decreases significantly to $36 \lambda / D$ compared to the apertures considered previously. At this small separation, diffraction effects from the off-axis star (which is not blocked by a coronograph) 


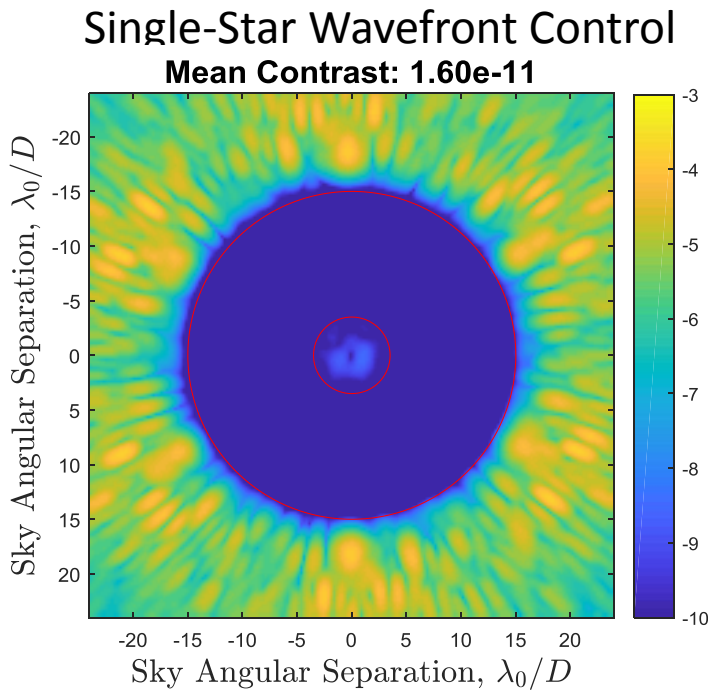

(a)

\section{Before Multi-Star Wavefront Control Mean Contrast: 2.20e-09}

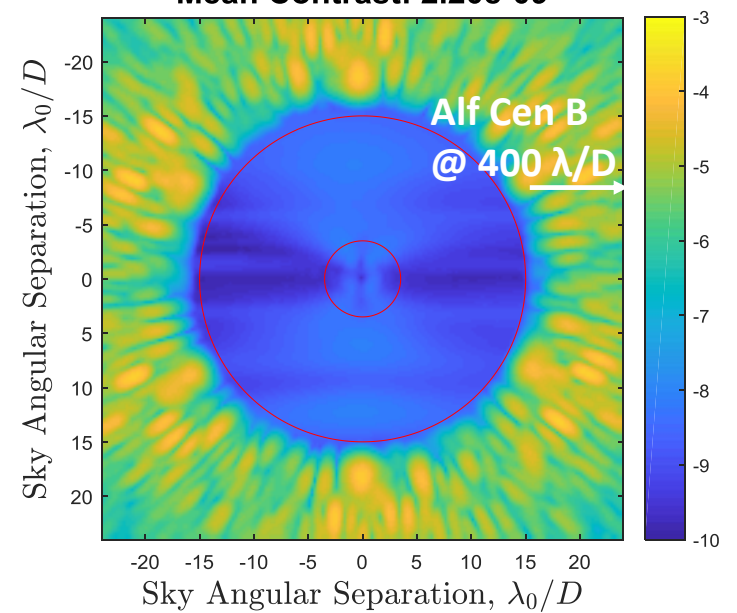

(b)

Figure 9: Baseline performance for the VVC-6 LUVOIR-B coronagraph in a 10\% band: (a) Single-star wavefront control showing a double-sided dark hole with contrast below the required 1e-10, (b) Keeping single-star wavefront control settings on the DM, but adding the off-axis source limits introduces a contrast floor due to the off-axis leakage at $2.20 \mathrm{e}-09$.

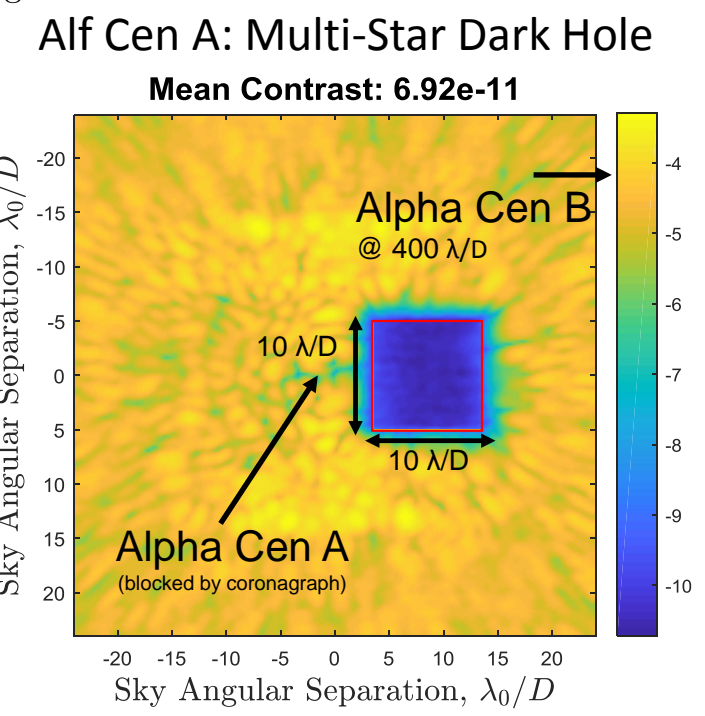

(a)

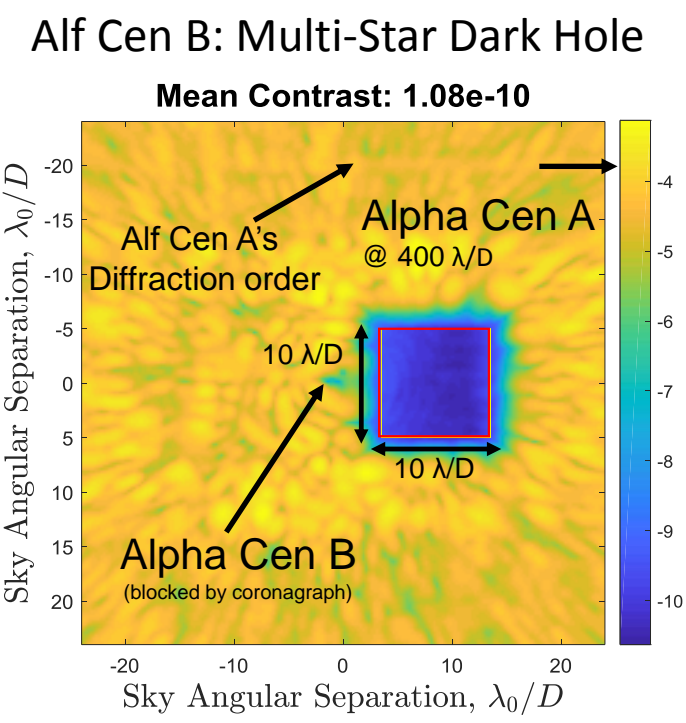

(b)

Figure 10: MSWC dark holes in 10\% band for with LUVOIR-B VVC-6 and with contrast within the 1e-10 target raw contrast when observing: (a) Alpha Centauri A, (b) Alpha Centauri B.

become more significant though even in this more extreme case the off-axis leakage is actually dominated by speckles induced by the primary PSD.

MSWC results are shown using the PIAA coronograph in Figure 11 with the on-axis and off-axis components correspondingly in Figure 11a and 11b. The PIAA coronagraph architecture includes an inverse PIAA set to recover a wide-field of view and increase PSF sharpenss. The first diffraction order as indicated in the off-axis contribution (in Figure 11b is used to control higher-frequency speckles outside the DM's nominal control limit for the off-axis star which is still outside the nominal Nyquist control limit for the smaller 32x32 DMs baselined.

Finally, MSWC is implemented with both PIAA and VVC-6 coronagraph configurations in Figure 12 demon- 
On-Axis Contribution

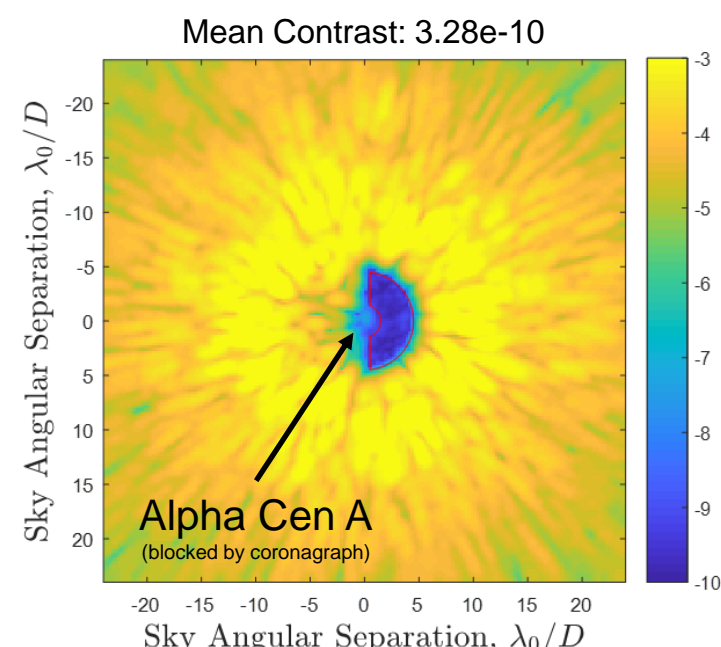

(a)

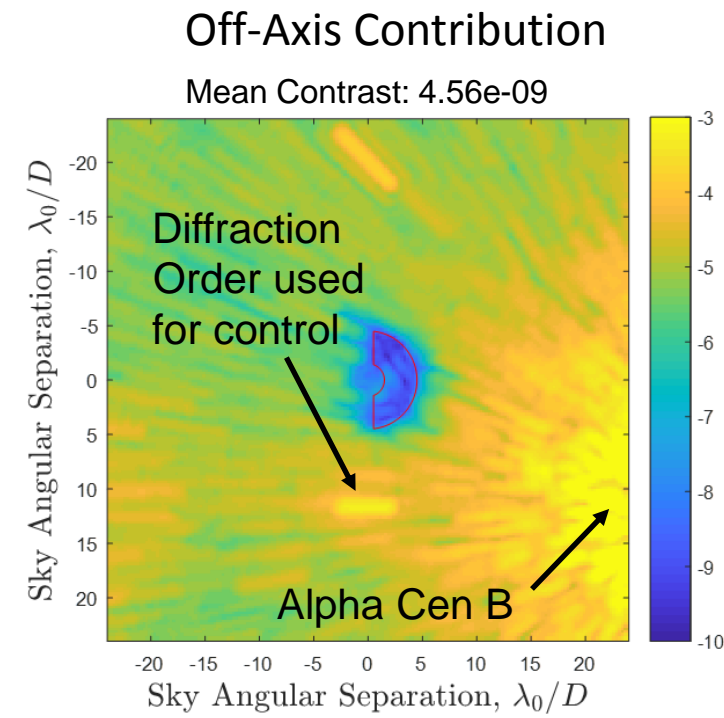

(b)

Figure 11: MSWC dark holes in 15\% band with ACEND for classical PIAA showing contributions from: (a) On-axis star, (b) Off-axis star.

\section{Multi-Star Dark Hole (PIAA)}

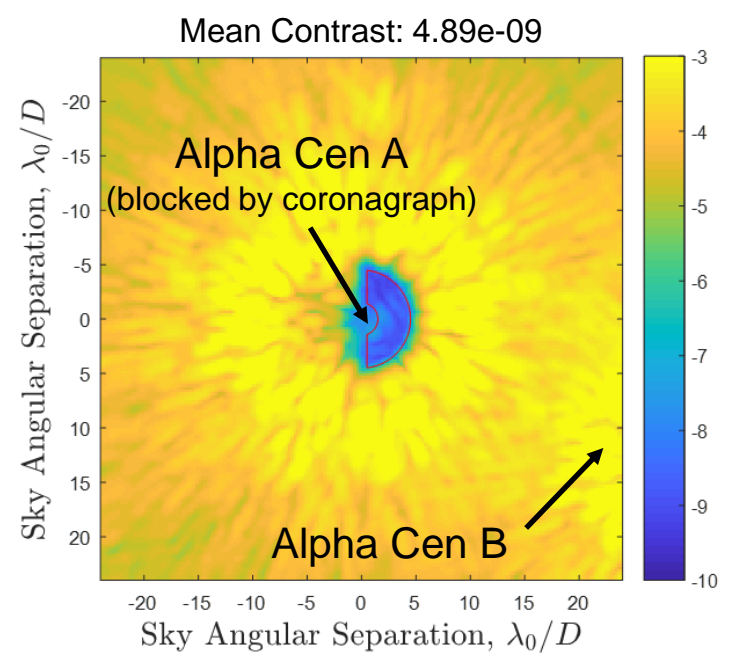

(a)

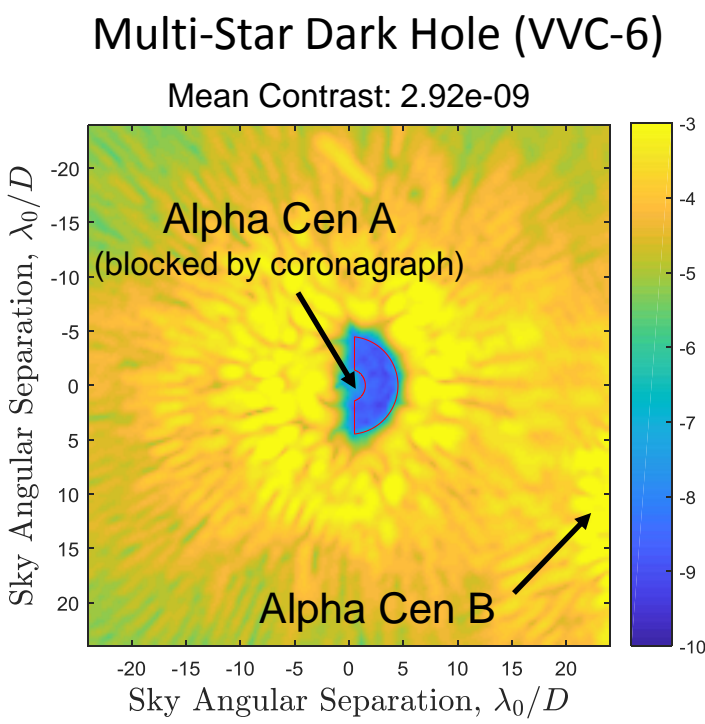

(b)

Figure 12: Comparison of MSWC dark holes in 15\% band for two different ACEND coronagraph architectures: (a) PIAA, (b) VVC6.

strating that both coronagraphs viable and very similar options in terms of performance and compatibility with MSWC.

\section{CONCLUSIONS}

This study has investigate the possibility of imaging multi-star systems with a variety of baselined coronagraphic instruments including WFIRST, Habex, LUVOIR. We have demonstrated the MSWC algorithm for a variety of mission configurations though simulated demonstrations using coronagraph layouts representative of each of these configurations and with realistic aberrations based on the official WFIRST primary PSD. For each case, we have first shown single-star performance at the required mission contrast levels. We have then used the Alpha Centauri stars as a representative geometry for a wide variety of binary stars, and have shown the contrast limits 
expected from the off-axis leakage introduced by the binary star companion.

In particular for the WFIRST CGI, we have extended on earlier studies which which have shown that the Wide-Field Imaging Mode is compatible with MSWC by improving performance under higher-fidelity conditions including a realistic model of the primary PSD aberations. Both the Alpha Centauri stars were demonstrated to the target raw contrast of $1 \mathrm{e}-9$ in $10 \%$ band for $10 \times 10 \lambda / D$ dark zones and we have also shown that wider bands including $15 \%$ are possible by trading off the size of the dark zone.

We have also shown through demonstrated simulations that MSWC is compatible with the VVC-6 for both the Habex-A (monolithic) and LUVOIR-B (off-axis, segmented) telescope pupils. For each of these cases, we have considered the actual Alpha Centauri stellar geometry and have demonstrated the feasibility of imaging both stars at the baseline contrast target level of 1e-10. Performance was very similar in both cases in spite of the LUVOIR-B pupil segmentation and the larger telescope pupil.

Finally, we have shown MSWC compatibility with an aperture as small as 0.4-m for both the PIAA and VVC6 configuration comparing an identical stellar geometry between for each of these two coronographs showing that a raw contrast close to $1 \mathrm{e}-9$ can be obtained.

We have explored a wide variety of coronographs (Shaped Pupils in Lyot configuration, Vector Vortex with a Charge-6, and PIAA) and with a telescope diameters ranging from 0.4-m to 8-m. All of these cases have been shown to be treatable with MSWC enabling binary star imaging and providing potential new targets and science for each of these missions primarily with an algorithmic implementation on the already baselined wavefront control loop.

\section{ACKNOWLEDGMENTS}

This work was supported in part by the National Aeronautics and Space Administration's Ames Research Center, as well as the NASA Technology Development for Exoplanet Missions (TDEM) component on Strategic Astrophysics Technology (SAT) through solicitation NNH16ZDA001N-SAT at NASA's Science Mission Directorate. Any opinions, findings, and conclusions or recommendations expressed in this article are those of the authors and do not necessarily reflect the views of the National Aeronautics and Space Administration. This research has made use of the Washington Double Star Catalog maintained at the U.S. Naval Observatory.

\section{REFERENCES}

1. D. Des Marais, M. Harwit, K. Jucks, J. Kasting, D. Lin, J. Kunine, J. Schneider, S. Seager, W. Traub, and N. Woolf, "Remote sensing of planetary properties and biosignatures on extrasolar terrestrial planets," Astrobiology 2, pp. 153-181, 2002.

2. S. Thomas, R. Belikov, and E. Bendek, "Techniques for High Contrast Imaging in Multi-Star Systems I: Super-Nyquist Wavefront Control," ApJ 810(1), 2015.

3. D. Sirbu, S. Thomas, R. Belikov, and E. Bendek, "Techniques for High Contrast Imaging in Multi-Star Systems II: Multi-Star Wavefront Control," ApJ 849(2), 2017.

4. D. Sirbu, R. Belikov, E. Bendek, E. Holte, A. J. E. Riggs, and S. B. Shaklan, "Prospects for exoplanet imaging in multi-star systems with starshades," Proc. of SPIE 104001D, 2017.

5. D. Sirbu, R. Belikov, E. Bendek, C. Henze, A. Riggs, and S. Shaklan, "Multi-star wavefront control for the wide-field infrared survey telescope," Proc. SPIE 106982F , 2018.

6. R. Belikov, E. Bendek, D. Sirbu, E. Pluzhnik, S. Bryson, et al., "Development of a method for exoplanet imaging in multi-star systems," NASA TDEM White Paper, 2018.

7. D. Raghavan et al., "A Survey of Stellar Families: Multiplicity of Solar-Type Stars," ApJS 190, 2010.

8. G. Duchêne and A. Kraus, "Stellar multiplicity," Annual Review of Astronomy and Astrophysics 51, 2013.

9. R. A. Matson, S. B. Howell, E. P. Horch, and M. E. Everett, "Stellar companions of exoplanet host stars in K2," AJ Accepted for publication, 2018.

10. B. Mason et al., "The 2001 US Naval Observatory Double Star CD-ROM. I. The Washington Double Star Catalog," $A J \mathbf{1 2 2}(6), 2001$. 
11. R. B. et al., "Direct imaging of exoplanets in nearby multi-star systems," Astro2020 Science White Paper 10400-30, 2019.

12. A. Give'on, R. Belikov, S. Shaklan, and N. Kasdin, "Closed-loop, dm diversity-based , wavefront correction algorithm for high-contrast imaging systems," Optics Express 15(19), 2007.

13. L. Pueyo, J. Kay, N. Kasdin, T. Groff, M. McElwain, A. Give'on, and R. Belikov, "Optimal dark hole generation via two deformable mirrors with stroke minimization," Applied Optics 48, 2009.

14. J. Krist, "Proper: an optical propagation library for idl," Proc. SPIE 6670P, 2007.

15. R. Juanola-Parramon, N. T. Zimmerman, and T. D. Groff, "Exoplanet detection with luvoir: evaluating the coronagraph sensitivity to telescope aberrations," Proc. SPIE 111172, 2019.

16. A. Riggs, N. T. Zimmerman, S. B. Shaklan, J. B. Jewell, and J. Gersh-Range, "Shaped pupil coronagraph design developments for the WFIRST coronagraph instrument," Proc. SPIE 10400-73, 2017.

17. D. Marx, E. Cady, A. J. E. Riggs, C. Prada, B. Kern, B.-J. Seo, and F. Shi, "Shaped pupil coronagraph: disk science mask experimental verification and testing," Proc. SPIE 1069849, 2018.

18. D. Sirbu, E. Pluzhnik, and R. Belikov, "Modeling of microelectromechanical systems deformable mirror diffraction grating," Proc. SPIE 990466, 2016.

19. O. Guyon, "Phase-induced amplitude apodization of telescope pupils for extrasolar terrestrial planet imaging," Astronomy and Astrophysics 404, 2003. 